\title{
Predicting necrosis in residual mass analysis after retroperitoneal lymph node dissection: a retrospective study
}

Eduardo de Paula Miranda ${ }^{1}$, Daniel Kanda Abe ${ }^{1}$, Adriano João Nesrallah', Sabrina Thalita dos Reis ${ }^{1}$, Alexandre Crippa', Miguel Srougi ${ }^{2}$ and Marcos Francisco Dall'Oglio ${ }^{1 *}$

\begin{abstract}
Background: Recent studies have demonstrated that pathological analysis of retroperitoneal residual masses of patients with testicular germ cell tumors revealed findings of necrotic debris or fibrosis in up to $50 \%$ of patients. We aimed at pursuing a clinical and pathological review of patients undergoing post chemotherapy retroperitoneal lymph node dissection (PC-RPLND) in order to identify variables that may help predict necrosis in the retroperitoneum.

Methods: We performed a retrospective analysis of all patients who underwent PC-RPLND at the University Hospital of the University of São Paulo and Cancer Institute of Sao Paulo between January 2005 and September 2011. Clinical and pathological data were obtained and consisted basically of: measures of retroperitoneal masses, histology of the orchiectomy specimen, serum tumor marker and retroperitoneal nodal size before and after chemotherapy.

Results: We gathered a total of 32 patients with a mean age of 29.7; pathological analysis in our series demonstrated that 15 (47\%) had necrosis in residual retroperitoneal masses, 15 had teratoma (47\%) and 2 (6.4\%) had viable germ cell tumors (GCT). The mean size of the retroperitoneal mass was $4.94 \mathrm{~cm}$ in our sample, without a difference between the groups $(P=0.176)$. From all studied variables, relative changes in retroperitoneal lymph node size $(P=0.04)$, the absence of teratoma in the orchiectomy specimen $(P=0.03)$ and the presence of choriocarcinoma in the testicular analysis after orchiectomy $(P=0.03)$ were statistically significant predictors of the presence of necrosis. A reduction level of $35 \%$ was therefore suggested to be the best cutoff for predicting the absence of tumor in the retroperitoneum with a sensitivity of $73.3 \%$ and specificity of $82.4 \%$.

Conclusions: Even though retroperitoneal lymph node dissection remains the gold standard for patients with residual masses, those without teratoma in the primary tumor and a shrinkage of 35\% or more in retroperitoneal mass have a considerably smaller chance of having viable GCT or teratoma in the retroperitoneum and a surveillance program could be considered.
\end{abstract}

Keywords: Testicular cancer, Retroperitoneal lymph node dissection, Necrosis, Teratoma

\footnotetext{
* Correspondence: marcosdallogliouro@terra.com.br

'Division of Urology, University of Sao Paulo Medical School and Cancer Institute of Sao Paulo, ICESP, São Paulo, SP, Brazil

Full list of author information is available at the end of the article
} 


\section{Background}

Testicular cancer has become one of the most curable solid neoplasms and serves as a paradigm for the multimodal treatment of malignancies. The appropriate integration of chemotherapy, retroperitoneal lymph node dissection (RPLND) and observation for the management of testis cancer has resulted in overall survival rates greater than $90 \%[1,2]$.

RPLND plays an important role in the management of patients with testicular germ cell tumors (GCTs), especially in those with residual masses after chemotherapy [3]. To date, series have demonstrated that pathological analysis of these masses reveals findings of necrotic debris or fibrosis in $40 \%$ to $50 \%$ of patients, teratoma in $35 \%$ to $40 \%$ and viable malignant cells in $10 \%$ to $15 \%$ of patients [4].

Even though early recognition and resection of teratoma in the retroperitoneum after chemotherapy have been accompanied by excellent prognosis, once it was initially thought to represent a benign course when present in the retroperitoneal space, but the untreated disease may have a lethal potential due to progressive local growth or malignant transformation, not to mention its classical unresponsiveness to conventional cisplatin-based chemotherapy regimens [3-6]. As for residual viable GCT, the consequence of its incomplete resection is certain disease progression [7]. Therefore, a more aggressive approach should always be considered when treating patients with teratoma or viable GCT in the retroperitoneum $[7,8]$.

Thus, the appropriate approach to residual masses following chemotherapy remains a controversial issue, since the literature has shown that as many as half of resected masses are basically composed of necrosis or fibrotic tissue and any sort of adjuvant therapy could be waived [9]. In order to avoid a great number of apparently unnecessary post-chemotherapy RPLND (PCRPLND), many studies have tried to develop algorithms to predict the presence of necrosis in the retroperitoneum. However, currently predictive models and imaging modalities cannot reliably predict the pathological finding of necrosis/fibrosis at PC-RPLND [9].

Some authors have established that patients without teratoma in the primary tumor and a shrinkage of $90 \%$ or more in retroperitoneal mass had little chance of having viable GCT or teratoma and could be safely put under a surveillance program with periodic imaging scans [10]. However, prospective analyses have demonstrated that approximately $30 \%$ of patients will harbor teratoma or viable malignancy even with normal postchemotherapy computed tomography (CT) results and no teratoma in the primary tumor [9].

The purpose of this work is to pursue a clinical and pathological review of patients undergoing PC-RPLND at a reference university oncology center in Brazil, in order to identify variables that may help predict the histological finding of necrosis in the retroperitoneum and perhaps establish a differentiated surveillance protocol.

\section{Methods}

We performed a retrospective analysis of all patients from our computerized database who underwent PCRPLND at our service between January 2005 and May 2011. Patients were operated on after having undergone three to four cycles of primary chemotherapy with bleomycin, etoposide and cisplatin.

Clinical and pathological data were obtained and consisted basically of measures of retroperitoneal masses, serum tumor markers, histology of the orchiectomy specimen, tumor marker values and retroperitoneal nodal size before and after chemotherapy.

The presence of either immature or mature teratoma in the resected specimen, as well as teratoma with malignant transformation, was considered part of the same group. Choriocarcinoma, yolk sac tumors, embryonal carcinoma and seminoma were considered viable GCTs. All histological findings were submitted to quantitative and qualitative analysis.

Post-chemotherapy alpha-fetoprotein (AFP) and lactate dehydrogenase (LDH) levels were registered as a continuous variable while human chorionic gonadotropin (hCG) levels were considered a categorical variable ranging from undetectable to detectable when serum concentrations were greater than $3 \mathrm{mIU}$.

Retroperitoneal nodal size before and after chemotherapy was determined by the longest transverse diameter of the largest mass on CT imaging. Relative change in nodal size before and after chemotherapy was calculated by dividing post-chemotherapy nodal size by pre-chemotherapy nodal size and was analyzed as a continuous variable.

Multiple variables were analyzed independently in order to establish any predictive value for finding necrotic tissue in the retroperitoneum. Statistical analysis was performed using the Statistical Package for Social Sciences (SPSS, version 12.0, Chicago, IL, USA), applying the Mann-Whitney $U$ test for non-parametric variables and the Fisher's exact test for categorical variables, with the level of significance set at $P<0.05$.

A cut-off level for predicting necrosis at retroperitoneal residual mass analysis was sought by constructing a receiver operating characteristic (ROC) curve of all significant variables, which were generated using graphical visualization in our statistical software. This study was carried-out in accordance with the Ethics Committee regulations.

\section{Results and discussion}

We gathered information on a total of 32 patients, who were 18- to 49-years old (mean age 30.5 ) and harbored 
seminomatous, nonseminomatous or mixed tumors in the testis tissue in different clinical stages. Three patients (9.4\%) had seminoma, five (16\%) had pure nonseminoma (choriocarcinoma, yolk sac tumors, embryonal carcinoma, teratoma, teratocarcinoma) and 24 (75\%) harbored mixed nonseminomatous GCT (NSGCT). None of the patients with seminoma had normal AFP levels, indicating that these patients may have had tumors with similar biological behavior to those with NSGCT.

At diagnosis, seven (22\%) patients were classified as having a stage I disease, while 21 (66\%) were stage II and four (12.5\%) were stage III. Clinical stage I patients were individuals with longer follow-up, who presented with retroperitoneal disease nonresponsive to chemotherapy and ultimately underwent PC-RPLND.

Pathological analysis in our series demonstrated that $15(47 \%)$ patients had necrosis in residual retroperitoneal masses, 15 had teratoma (47\%) and two (6\%) had viable GCT: one seminoma and one yolk sac tumor. For statistical reasons and in alignment with the aims of this study, we divided those patients into two groups, assembling patients with viable GCT and teratoma and analyzing them as one group. Mean size of the retroperitoneal mass was $4.94 \mathrm{~cm}$ in our sample, $3.79 \mathrm{~cm}$ in the group of necrosis and $5.96 \mathrm{~cm}$ in the group of teratoma and viable GCT $(P=0.176)$. There was also no difference between groups regarding stratification of nodal size, as shown in Table 1.

Primary tumor histology revealed embryonal cell carcinoma in $56 \%$, seminomatous elements in $16 \%$, yolk sac tumor in $19 \%$ and teratomatous elements in $56 \%$ of the patients. Of the 18 patients with teratomatous elements in the primary tumor, 13 (72\%) had teratoma in the retroperitoneum at PC-RPLND. Even in the absence of teratoma in the primary tumor, teratoma was present in the retroperitoneum in five patients (16\%).

When comparing pathological analysis of primary tumor specimens, we found a statistical difference when comparing the prevalence of teratoma and choriocarcinoma.

Table 1 Distribution according to nodal size in men undergoing PC-RPLND

\begin{tabular}{lcccc}
\hline & Necrosis & $\begin{array}{c}\text { Teratoma or } \\
\text { Viable GCT }\end{array}$ & Total & $\begin{array}{c}\boldsymbol{P} \\
\text { Value }\end{array}$ \\
\hline Number of patients (\%) & $15(47)$ & $17(53)$ & 32 & \\
Mean size $(\mathrm{cm})$ & 3.79 & 5.96 & 4.94 & 0.176 \\
Node size number (\%) & $1(7)$ & $1(6)$ & $2(6)$ & 0.514 \\
$1 \mathrm{~cm}$ or less & $3(20.0)$ & $0(0.0)$ & $3(10)$ & 0.091 \\
1 to $2 \mathrm{~cm}$ & $8(53)$ & $9(53)$ & $17(53)$ & 0.630 \\
2 to $5 \mathrm{~cm}$ & $3(20.0)$ & $7(41)$ & $10(31)$ & 0.182 \\
Greater than $5 \mathrm{~cm}$ & & & & \\
\hline
\end{tabular}

Data are shown as number (\%). GCT, germ cell tumor; PC-RPLND, postchemotherapy retroperitoneal lymph node dissection.
Other findings, such as the presence of seminoma, yolk sac tumor, embryonal carcinoma, endodermal sinus tumor lymph vascular invasion (LVI), rete testis invasion and spermatic invasion were similar between groups.

Univariate analysis of quantitative components at orchiectomy histology, retroperitoneal node size with its relative measures and serum markers are shown in Table 2.

Even though the presence of teratoma in the primary tumor was an important negative predictor for the finding of necrosis, the quantitative analysis has proven to be statistically irrelevant.

While relative reduction in mass size after chemotherapy has been shown to be an important predictor of necrosis, even when considering patients not responding to chemotherapy, absolute reduction and enlargement of the residual mass did not show a significant difference between groups.

There was a statistical difference in AFP levels between groups; however, when comparing relative changes in AFP levels after chemotherapy, no difference was found. Comparison of LDH levels and their relative changes was

Table 2 Univariate analysis predicting necrosis at PCRPLND

\begin{tabular}{|c|c|c|c|}
\hline & Necrosis & $\begin{array}{l}\text { Teratoma or } \\
\text { viable GCT }\end{array}$ & $\begin{array}{c}P \\
\text { Value }\end{array}$ \\
\hline \multicolumn{4}{|l|}{$\begin{array}{l}\text { Components at orchiectomy } \\
\text { histology (\%): }\end{array}$} \\
\hline Embryonal & $22.7 \pm 30.1$ & $32.9 \pm 29.7$ & 0.370 \\
\hline Yolk sac & $4.3 \pm 11.5$ & $4.1 \pm 8.0$ & 0.737 \\
\hline Teratoma & $12.7 \pm 23.7$ & $13.8 \pm 17.1$ & 0.502 \\
\hline Endodermal sinus & $1.7 \pm 4.5$ & $5.0 \pm 11.7$ & 0.628 \\
\hline \multicolumn{4}{|l|}{ Retroperitonial node size } \\
\hline RP node $\mathrm{cm}$ before chemotherapy & $6.5 \pm 4.1$ & $6.1 \pm 5.5$ & 0.849 \\
\hline $\mathrm{RP}$ node $\mathrm{cm}$ after chemotherapy & $3.2 \pm 1.8$ & $5.3 \pm 4.8$ & 0.331 \\
\hline Relative change in RP node size & $31.9 \pm 48.3$ & $9.4 \pm 38.8$ & 0.044 \\
\hline Absolute change in RP node size & $3.2 \pm 2.9$ & $0.78 \pm 2.9$ & 0.053 \\
\hline Relative reduction in RP node & $56.4 \pm 14.0$ & $26.2 \pm 20.1$ & $<0.001$ \\
\hline Relative enlargement in RP node & $35.6 \pm 44.1$ & $60.1 \pm 22.5$ & 0.425 \\
\hline \multicolumn{4}{|l|}{ Serum markers } \\
\hline hCG after chemotherapy & 0.0 & 0.0 & $* *$ \\
\hline AFP after chemotherapy & $1.3 \pm 0.4$ & $22.1 \pm 49.3$ & $<0.001$ \\
\hline $\begin{array}{l}\text { Relative change in AFP after } \\
\text { chemotherapy }\end{array}$ & $15.7 \pm 46.7$ & $-83.7 \pm 5.13$ & 0.075 \\
\hline LDH after chemotherapy & $307.7 \pm 60.7$ & $324.0 \pm 65.8$ & 0.399 \\
\hline $\begin{array}{l}\text { Relative change in LDH after } \\
\text { chemotherapy }\end{array}$ & $1.5 \pm 15.9$ & $0.68 \pm 27.4$ & 0.915 \\
\hline
\end{tabular}

** unable to calculate. Data expressed as mean \pm standard deviation. AFP, alpha-fetoprotein; GCT, germ cell tumor; hCG, human chorionic gonadotropin; $\mathrm{LDH}$, lactate dehydrogenase; PC-RPLND, post-chemotherapy retroperitoneal lymph node dissection; RP, retroperitoneal. 
nonsignificant. We were unable to compare hCG levels between groups because we only had two patients whose hCG levels were not undetectable.

Of all the studied variables, relative changes in retroperitoneal lymph node size $(P=0.04)$, the absence of teratoma in the orchiectomy specimen $(P=0.03)$ and the presence of choriocarcinoma in the testicular analysis after orchiectomy ( $P=0.03)$ were statistically significant predictors of the presence of necrosis in the retroperitoneum.

ROC curves were built for variables that were independent predictors of necrosis at PC-RPLND. Even though the size of the retroperitoneal mass after chemotherapy showed no statistical difference, we also built a curve for it. The variable relative reduction after chemotherapy was the only one with predictive value according to the Youlden index. An area under the curve (AUC) of 0.710 was obtained and a reduction level of $35 \%$ was therefore suggested to be the best cutoff for predicting the absence of tumor in the retroperitoneum with a sensitivity of $73 \%$ and a specificity of $82 \%$. ROC curves of isolated size of retroperitoneal mass, absence of teratoma and presence of choriocarcinoma did not indicate significant findings.

In our institution, relative change in retroperitoneal node size, absence of teratoma in the orchiectomy specimen and the presence of choriocarcinoma in the testicular analysis after orchiectomy were statistically significant predictors of the presence of necrosis in the retroperitoneum and a surveillance program could be considered, given the uncertainty in predicting the histology of residual masses after chemotherapy in patients with metastatic testicular tumors.

Analysis of residual retroperitoneal masses after chemotherapy is being increasingly regarded as a fundamental issue, not only for orienting adjuvant therapies, but also because it has prognostic implications [11]. Outcome assessments in patients with NSGCT have demonstrated that incomplete resection of residual retroperitoneal masses, the size of residual retroperitoneal masses and the finding of teratoma and viable GCT at RPLND independently predict disease progression and relapse $[11,12]$.

The size of residual retroperitoneal masses after chemotherapy has traditionally been considered when choosing the subsequent treatment modality [9]. Studies have shown that residual masses smaller than $2 \mathrm{~cm}$ are considered one of the most significant predictors for finding necrosis at PC-RPLND at logistic regression [4]. Furthermore, a number of investigators continue to base the decision to perform PC-RPLND on residual mass size alone, obviating PC-RPLND in patients with residual masses of $1 \mathrm{~cm}$ or less [13].

However, recent studies have shown that after chemotherapy a third of retroperitoneal masses of $<2 \mathrm{~cm}$ harbored either teratoma or viable GCT [14]. Even though we found a trend of having only necrosis in the retroperitoneum for masses $<2 \mathrm{~cm}(P=0.09)$, in the present study residual mass size alone has not appeared to be a good predictor of necrosis, with nonsignificant size differences between groups and an underrated AUC in ROC curves. In addition, one case of teratoma in a $1 \mathrm{~cm}$ mass was registered in our sample. Therefore, we advocate that the decision of not operating on patients cannot be based on mass size alone due to the lack of consensus in the literature $[9,10,13,14]$.

Traditional series report necrotic debris or fibrosis in $40 \%$ to $50 \%$, teratoma in $35 \%$ to $40 \%$ and viable malignant cells in $10 \%$ to $15 \%$ of patients [3]. In more recent analyses, the incidence of residual microscopic teratoma in the retroperitoneum has decreased to approximately $20 \%$ to $25 \%$, with an increase of necrotic tissue findings of up to $60 \%$ and stable rates of viable GCT [15]. It has been reported that an increase in the proportion of necrosis is generally attributed to stage migration and the use of more effective chemotherapy regimens, especially in patients achieving a complete response to chemotherapy [15].

In our series we found a distribution pattern similar to former studies, with almost $50 \%$ of patients harboring teratoma in the retroperitoneal histology at RPLND, which may suggest that our chemotherapy regimens have been having inferior rates of complete responders or simply because our group is composed of patients in more advanced stages.

Teratoma-negative primary tumor and volumetric regression of at least $90 \%$ after chemotherapy have been described as being highly predictive of harboring necrosis only at PC-RPLND $[9,16]$. Other series include normal serum tumor markers, and node size $<2 \mathrm{~cm}$, the presence of yolk sac tumor or embryonal carcinoma on primary tumor and lymph vascular invasion, among others $[9,10,15-17]$. On the other hand, some authors have been unable to identify variables to be highly predictive of harboring necrosis only at PC-RPLND $[15,18]$. Nevertheless, currently predictive models fail to accurately predict necrosis in the retroperitoneum, since almost $30 \%$ of patients will harbor teratoma or viable malignancy even with normal post-chemotherapy CT and no teratoma in the primary tumor [9]. A study carried out at the Memorial Sloan-Kettering Cancer Center revealed that $26 \%$ of patients had teratoma or viable GCT, even those with radiographically normal retroperitoneum who underwent PC-RPLND [9].

The relatively small number of patients when compared to other institutional retrospective studies is a limitation of the present study; however, other studies with similar sample sizes have also come to significant findings. 
In the present study, relative changes in retroperitoneal node size stood out as the single best predictor of the presence of necrosis in the retroperitoneum, which is in accordance with actual logistic regression models $[9,10]$. While other series suggest $90 \%$ as a shrinkage cutoff value to reliably predict necrosis, our quantitative analysis have demonstrated a cutoff level of 35\% with approximate sensitivity and specificity [9].

The absence of teratoma has also been demonstrated to be significant in qualitative analysis, but not in quantitative analysis. The presence of choriocarcinoma in the testicular histology predicted necrosis in the retroperitoneum, despite only four (12.5\%) patients who had such a finding. This association is an interesting finding that has not been previously reported. We reviewed the literature and no possible explanations were found. We believe further investigation is necessary to confirm this finding, since the number of patients in our sample is relatively small.

\section{Conclusions}

Even though RPLND remains the gold standard for patients with residual masses, those without teratoma in the primary tumor and a shrinkage of $35 \%$ or more in retroperitoneal mass have a considerably smaller chance of having viable GCTs or teratoma in the retroperitoneum.

\section{Abbreviations \\ AFP: Alphs-fetoprotein; AUC: Area under the curve; CT: Computed tomography; GCT: Germ cell tumor; hCG: Human chorionic gonadotropin; LDH: Lactate dehydrogenase; LVI: Lymph vascular invasion; NSGCT: Nonseminomatous germ cell tumor; PC-RPLND: Post chemotherapy retroperitoneal lymph node dissection; ROC: Receiver operating characteristic; RPLND: Retroperitoneal lymph node dissection.}

\section{Competing interests}

The authors declare that they have no competing interests.

\section{Authors' contributions}

EPM and DKA gathered and compiled data and have been involved in drafting the manuscript. AJN and STL participated in the design of the study and performed the statistical analysis. ACS and MS revised the manuscript critically for important intellectual content. MS and MFD have given final approval of the version to be published. All authors read and approved the final manuscript.

\section{Author details}

'Division of Urology, University of Sao Paulo Medical School and Cancer Institute of Sao Paulo, ICESP, São Paulo, SP, Brazil. ²Division of Urology, University of São Paulo Medical School, São Paulo, Brazil.

Received: 29 November 2011 Accepted: 31 August 2012 Published: 28 September 2012

\section{References}

1. Foster R, Bihrle R: Current status of retroperitoneal lymph node dissection and testicular cancer: when to operate. Cancer Control 2002, 9:277-283.

2. Dearnaley $D$, Huddart $R$, Horwich A: Regular review: managing testicular cancer. BMJ 2001, 322:1583-1588.

3. Luz AM, Kotb AF, Aldousari S, Brimo F, Tanguay S, Kassouf W, Aprikian AG: Retroperitoneal lymph node dissection for residual masses after chemotherapy in nonseminomatous germ cell testicular tumor. World 」 Surg Oncol 2010, 8:97.
4. Steyerberg EW, Keizer HJ, Fossa SD, Sleijfer DT, Toner GC, SchraffordtKoops H, Mulders PF, Messemer JE, Ney K, Donohue JP, et al: Prediction of residual retroperitoneal mass histology after chemotherapy for metastatic nonseminomatous germ cell tumor: multivariate analysis of individual patient data from six study groups. J Clin Oncol 1995, 13:1177-1187.

5. Lorigan JG, Eftekhari F, David CL, Shirkhoda A: The growing teratoma syndrome: an unusual manifestation of treated, nonseminomatous germ cell tumors of the testis. AJR Am J Roentgenol 1988, 151:325-329.

6. Tait D, Peckham MJ, Hendry WF, Goldstraw P: Post-chemotherapy surgery in advanced non-seminomatous germ-cell testicular tumours: the significance of histology with particular reference to differentiated (mature) teratoma. Br J Cancer 1984, 50:601-609.

7. Fizazi K, Tjulandin S, Salvioni R, Germà-Lluch JR, Bouzy J, Ragan D, Bokemeyer C, Gerl A, Fléchon A, de Bono JS, Stenning S, Horwich A, Pont J, Albers P, De Giorgi U, Bower M, Bulanov A, Pizzocaro G, Aparicio J, Nichols CR, Théodore C, Hartmann JT, Schmoll HJ, Kaye SB, Culine S, Droz JP, Mahé $\mathrm{C}$ : Viable malignant cells after primary chemotherapy for disseminated nonseminomatous germ cell tumors: prognostic factors and role of postsurgery chemotherapy-results from an international study group. J Clin Oncol 2001, 19:2647-2657.

8. Motzer RJ, Amsterdam A, Prieto V, Sheinfeld J, Murty W, Mazumdar M, Bosl GJ, Chaganti RS, Reuter VE: Teratoma with malignant transformation: diverse malignant histologies arising in men with germ cell tumors. J Urol 1998, 159:133-138.

9. Carver BS, Bianco FJ Jr, Shayegan B, Vickers A, Motzer RJ, Bosl GJ, Sheinfeld $\mathrm{J}$ : Predicting teratoma in the retroperitoneum in men undergoing postchemotherapy retroperitoneal lymph node dissection. J Urol 2006 176:100-103.

10. El Sayed S, Grando JP, Almeida SH, MortatiNeto N, Moreira HA: Postchemotherapy residual mass in non-seminomatous testicular cancer. The role of retroperitoneal lymph node dissection. Int Braz J Urol 2004, 30:384-388.

11. Shayegan B, Carver BS, Stasi J, Motzer RJ, Bosl GJ, Sheinfeld J: Clinical outcome following post-chemotherapy retroperitoneal lymph node dissection in men with intermediate- and poor-risk nonseminomatous germ cell tumour. BJU Int 2007, 99:993-997.

12. Sheinfeld J: The role of adjunctive postchemotherapy surgery for nonseminomatous germ-cell tumors: current concepts and controversies. Semin Urol Oncol 2002, 20:262-271.

13. Schmoll HJ, Souchon R, Krege S, Albers P, Beyer J, Kollmannsberger C, Fossa SD, Skakkebaek NE, de Wit R, Fizazi K, Droz JP, Pizzocaro G, Daugaard G, de Mulder PH, Horwich A, Oliver T, Huddart R, Rosti G, Paz Ares L, Pont O, Hartmann JT, Aass N, Algaba F, Bamberg M, Bodrogi I, Bokemeyer C, Classen J, Clemm S, Culine S, de Wit M, et al: European consensus on diagnosis and treatment of germ cell cancer: a report of the European germ cell cancer consensus group (EGCCCG). Ann Oncol 2004, 15:1377-1399.

14. Oldenburg J, Alfsen GC, Lien HH, Aass N, Waehre H, Fossa SD: Postchemotherapy retroperitoneal surgery remains necessary in patients with nonseminomatous testicular cancer and minimal residual tumor masses. J Clin Oncol 2003, 21:3310-3317.

15. Beck SD, Foster RS: Management of the post chemotherapy subcentimeter residual mass: the case for observation. World J Urol 2009, 27:485-488.

16. Donohue JP, Rowland RG, Kopecky K, Steidle CP, Geier G, Ney KG, Einhorn L, Williams S, Loehrer P: Correlation of computerized tomographic changes and histological findings in 80 patients having radical retroperitoneal lymph node dissection after chemotherapy for testis cancer. J Urol 1987 137:1176-1179.

17. Nicolai N, Miceli R, Artusi R, Piva L, Pizzocaro G, Salvioni R: A simple model for predicting nodal metastasis in patients with clinical stage I nonseminomatous germ cell testicular tumors undergoing retroperitoneal lymph node dissection only. J Urol 2004, 171:172-176.

18. Fossa SD, Qvist H, Stenwig AE, Lien HH, Ous S, Giercksky KE: Is postchemotherapy retroperitoneal surgery necessary in patients with nonseminomatous testicular cancer and minimal residual tumor masses? J Clin Oncol 1992, 10:569-573.

\section{doi:10.1186/1477-7819-10-203}

Cite this article as: Miranda et al:: Predicting necrosis in residual mass analysis after retroperitoneal lymph node dissection: a retrospective study. World Journal of Surgical Oncology 2012 10:203. 